\title{
Experimental Evaluation of a Hyperbolic Ion Trap for Fourier Transform Ion Cyclotron Resonance Mass Spectrometry
}

\author{
Winnie W. Yin, Mingda Wang, ${ }^{*}$ and Alan G. Marshall ${ }^{\dagger}$ \\ Department of Chemistry, The Ohio State University, Columbus, Ohio, USA
}

Edward B. Ledford, Jr.

ICR Research Associates, Inc., Lincoln, Nebraska, USA

The Penning ion trap, consisting of hyperbolically curved electrodes arranged as an unbroken ring electrode capped by two end electrodes whose interelectrode axis lies along the direction of an applied static magnetic feld, has long been used for single-ion trapping. More recently, it has been used in "parametric" mode for ion cyclotron resonance (ICR) detection of off-axis ions. In this article, we describe and test a Penning trap whose ring electrode has been cut into four equal quadrants for conventional dipolar ICR excitation (on one pair of opposed ring quadrants) and dipolar ICR detection (on the other pair). In direct comparisons to a cubic trap, the present hyperbolic trap offers somewhat improved ICR mass spectral peak shape, higher mass resolving power, and comparable frequency shift as a function of trapping voltage. Mass measurement accuracy over a wide mass range is improved twofold and mass discrimination is somewhat worse than for a cubic trap. The relative advantages of parametric, dipolar, and quadrupole modes are briefly discussed in comparison to screened and unscreened cubic traps. (J Am Soc Mass Spectrom 1992, 3, 188-197)

I Fourier transform ion cyclotron resonance (FT/ICR) mass spectrometry [1-16], ions are typically trapped by static magnetic and electric fields produced by ion traps of various geometry (see Figure 1a-d). An ion moving in spatially uniform electric and magnetic fields, $E$ and $B$, is subject to a "Lorentz" force,

Force $=$ mass $\cdot$ acceleration

$$
\begin{aligned}
& =\text { mass } \cdot \text { acceleration } \\
& =m d v / d t=q E+q v \times B \quad \text { (SI units) }
\end{aligned}
$$

in which $m, q$, and $v$ are the mass, charge, and velocity of the ion. In the limit that the electric field is zero everywhere inside the ion trap, the trajectory of the ion in the $x-y$ plane perpendicular to the applied magnetic field direction follows a circular orbit with a natural "cyclotron" orbital frequency, $\omega_{\mathrm{c}}\left(\mathrm{rad} \mathrm{s}^{-1}\right)$,

$$
\omega_{\mathrm{c}}=\frac{q B}{m}
$$

in which $\omega_{c}=2 \pi v_{c}$, with $\nu_{c}$ in $\mathrm{Hz}$.

*Current address: Walnut Creek Instrument Division, Varian Associates, 2700 Mitchell Drive, P.O. Box 9016, Walnut Creek, CA 94598 . 0916 .

$\dagger$ Also a member of the Department of Biochemistry.

Address reprint request to Alan G. Marshall, Department of Chemistry, The Ohio State University, 120 West 18th Avenue, Columbus, OH 43210.
Cubic, Tetragonal, and Cylindrical Ion Traps

Although a static magnetic field applied along the $z$-direction constrains ion motion in the $x$-y plane, ions are otherwise free to move along the magnetic field direction (z-axis). Therefore, an additional electrostatic "trapping" potential, produced by applying a small static voltage $(-1 \mathrm{~V})$ to each of two "end

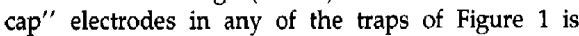
needed to provide a restoring $z$-force to prevent ions from escaping along the $z$-direction. The observed ion cyclotron frequency necessarily then differs from the unperturbed cyclotron orbital frequency, $\omega_{c}$, since $E$ in eq 1 is no longer spatially uniform.

Most early FT/ICR mass spectrometry experiments were performed with a cubic ICR ion trap [17] shown in Figure 1a. The structural simplicity of the cubic trap (and the closely related orthorhombic [18] and tetragonal [19] traps) has led to their widespread use in FT/ICR mass spectrometry. Another popular design (Figure 1b) is the right circular cylindrical trap [20] first reduced to practice as an unbroken cylindrical ring electrode with off-axis ion injection [21], and now typically operated with on-axis ions in a cylinder cut into four equal [22] or unequal [23] quadrants. The excitation [24] and detection [25] properties of cubic, 
(a)

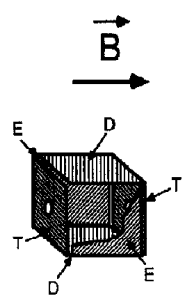

(c)

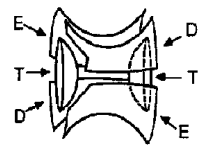

(b)
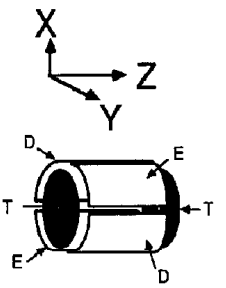

(d)

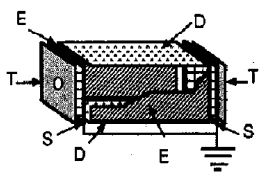

Figure 1. Static electromagnetic ion traps for FT/ICR mașs spectrometry. $B_{0}$ denotes the (static) magnetic field. The excitation, detection, and trap electrodes are designated by $E, D$, and $T$, respectively. (a) Cubic; (b) cylindrical; (c) hyperbolic; (d) screened cubic.

tetragonal, and cylindrical traps have recently been summarized, All such traps exhibit an approximately quadrupolar trapping potential (see below) near the center of the trap. Detection sensitivity for tetragonal and cylindrical traps of the same aspect (i.e., length to width) ratio is comparable [25], and both kinds of traps exhibit a mass-independent (but trapping voltage-dependent) shift in ICR orbital frequency. The trapping shift may be reduced $[26,27]$ or effectively eliminated [28] by segmenting $[26,27]$ or screening (Figure 1d) [28] the trapping electrodes. Finally, spatial inhomogeneity in the radiofrequency excitation field may be effectively eliminated by introduction of "shim" or "guard" wires to which appropriately divided if voltages are applied [29-31].

\section{Quadrupolar Ion Trap}

The desirability of a quadrupolar electrostatic trapping potential is evident from the following simple (partial) analysis of eq 1 . If we consider motion in the $z=0$ midplane of the trap, for an ion with $x y$-velocity, $v_{x y}$, with cyclotron orbit centered on the z-axis, and zero $z$-velocity, then the scalar form of eq 1 reduces to:

$$
\frac{m v_{x y}^{2}}{r}=q E(x, y)+q v_{x y} B
$$

Thus, if we could produce a static electric field which increases as the distance, $r=\sqrt{x^{2}+y^{2}}$, from the $z$-axis, then eq 3 would take the form,

$$
\frac{m v_{x y}^{2}}{r}=-q E_{0} r+q v_{x y} B
$$

in which $E=-E_{0} r$ is the (radially outward-directed, hence negative sign) electric field at radius $r$. Because

angular velocity, $\omega=v_{x y} / r$, eq 4 then reduces to

$$
m \omega^{2} r=-q E_{0} r+q \omega r B
$$

or

$$
\omega^{2}-\frac{q B}{m} \omega+\frac{q E_{0}}{m}=0
$$

The two roots, $\omega_{+}$and $\omega_{-}$, of the quadratic eq 5 represent the ICR "orbital" and "magnetron" frequencies. Although the orbital frequency is no longer simply $q B / m$, the orbital frequency is nevertheless clearly independent of radius, a key property that we shall discuss further below.

The highly desirable independence of ICR orbital frequency on radius requires an electrostatic field which is proportional to radius $r$. Since electric field is the negative gradient of electrostatic potential, it is clear that the electrostatic radial potential, $U(x, y, z)$, must vary as $r^{2}$. However, Laplace's equation,

$$
\begin{aligned}
\nabla^{2} U(x, y, z)= & \frac{\partial^{2} U(x, y, z)}{\partial x^{2}}+\frac{\partial^{2} U(x, y, z)}{\partial y^{2}} \\
& +\frac{\partial^{2} U(x, y, z)}{\partial z^{2}}=0
\end{aligned}
$$

requires that the potential must vary as $z^{2}$ as well The resulting "quadrupolar" potential has the form [32],

$$
U(x, y, z)=V_{\mathrm{T}} \frac{r_{0}^{2}-r^{2}+2 z^{2}}{r_{0}^{2}+2 z_{0}^{2}}
$$

in which $V_{\mathrm{T}}$ is a "trapping" potential difference, and $r_{0}$ and $z_{0}$ are characteristic trap dimensions (see below). Equation 7 clearly satisfies eq 6 .

\section{Hyperbolic Ion Trap}

The remaining question is how to produce the quadrupolar potential of eq 7. The answer is simply to fabricate three electrodes, each of whose surfaces follows the (hyperbolic) equipotential surfaces of eq 7 (see Figures 1 and 2). The "ring" electrode follows the hyperbola of revolution [32],

$$
z^{2}=\frac{1}{2}\left(r^{2}-r_{0}^{2}\right)
$$

and two "end cap" electrodes follow the two hyperbolas of revolution (one for positive $z$-values and one for negative $z$-values, with $z=0$ at the center of the trap),

$$
z^{2}=z_{0}^{2}+\frac{r^{2}}{2}
$$


in which $r_{0}$ and $z_{0}$ are the minimum distances from the center of the trap to the ring and end cap electrodes. Finally, in order to operate such a trap with the usual dipolar excitation [17, 24] (produced by applying an if voltage differentially across two opposed transmitter electrodes) and dipolar detection $[17,25]$ (i.e., measurement of the difference in induced charge between two opposed detector electrodes [25, 33], we cut the ring electrode into four equal quadrants (see Figure 2), as first proposed and demonstrated for single-mass measurements [34]. Other operating modes (parametric and quadrupole) will be discussed at the end of this article.

As noted above, the electrostatic field inside a cubic, tetragonal, or cylindrical trap deviates increasingly from the quadrupolar form with increasing distance away from the center of the trap. As a result, the ICR orbital frequency varies with $r$ and $z$. In contrast, ICR orbital frequency in an infinitely extended hyperbolic trap has the same value anywhere inside the region bounded by the three electrodes (eq $8 \mathrm{a}$ and $\mathrm{b}$ ). The cyclotron orbital and magnetron frequencies, $\omega_{+}$and $\omega_{-}$take the form,

$$
\begin{aligned}
& \omega_{+}=\frac{1}{2}\left(\omega_{c}+\omega_{c} \sqrt{1-\frac{m}{m_{\text {crit }}}}\right) \\
& \omega_{-}=\frac{1}{2}\left(\omega_{c}-\omega_{c} \sqrt{1-\frac{m}{m_{\text {crit }}}}\right)
\end{aligned}
$$

in which

$$
m_{\text {crit }}=\frac{q B^{2}\left(r_{0}^{2}+2 z_{0}^{2}\right)}{8 V_{T}}
$$

In this article, we report a series of experimental performance tests of a hyperbolic ion trap for FT/ICR mass spectrometry: (1) effect of trapping voltage on ICR orbital frequency; (2) effect of rf electric field excitation amplitude and duration on ICR orbital frequency, ion ejection, and magnitude-mode FT/ICR peak shape; (3) effect of number of formed and trapped ions on ICR orbital frequency and FT/ICR spectral peak height; and (4) accurate mass measurement capability. In each case, the hyperbolic trap performance is compared to that of a cubic trap of similar dimensions.

\section{Experimental}

An ion trap with two hyperbolic end caps $\left(z_{0}=0.250\right.$ in. $=6.35 \times 10^{-3} \mathrm{~m}$ ) and a hyperbolic ring electrode ( $r_{0}=0.500$ in. $=1.27 \times 10^{-2} \mathrm{~m}$ ) split into four equal quadrants was fabricated from solid oxygen-free hard copper expressly for this study (Figure 2) [35]. The trap was designed to have a one-inch "waist" (i.e., 1-in. minimum diameter of the ring electrode), because the maximum outer diameter of the ring elec-

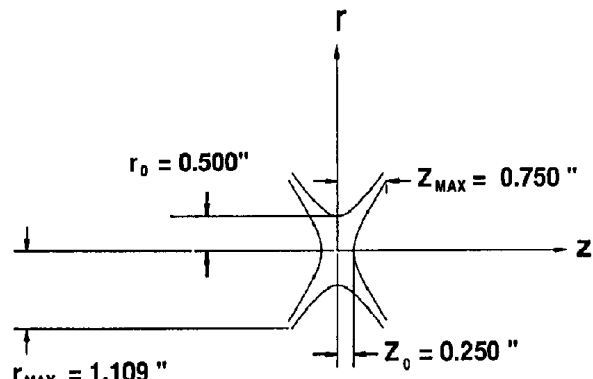

$r_{\text {max }}=1.109$ "

Figure 2. Cross-section through the $x z$ plane $(y=0)$ of the hyperbolic ion trap used in this work. This trap produces a near-perfect quadrupolar electrostatic potential. Note that $r_{0}=$ $2 z_{0}$ (see text)

trode and its supports had to be slightly less than the 4-in. diameter of the vacuum chamber within the superconducting magnet bore. The trap segments were held apart in the $z$-direction by sapphire bearings. Then 2-mm diameter holes were bored along the $z$-axis to provide for introduction of an electron beam from a rhenium filament mounted about $1 \mathrm{~cm}$ outside one end of the trap. FT/ICR mass spectra were generated with an Extrel ANS (Madison, WI) FTMS-1000 instrument operating at an applied magnetic field induction of $3.0 \mathrm{~T}$. Gaseous samples were introduced through a Varian Associates (Walnut Creek, CA) No. 951-5100 leak valve. A 1-in. cubic trap was substituted on the same instrument for comparison.

For determination of the shift in ICR orbital frequency of $\mathrm{C}_{6} \mathrm{H}_{6}^{+}$as a function of trapping voltage, we excited each time domain signal by applying singlefrequency rf excitation $\left(23.83 \mathrm{~V}_{(\mathrm{p}-\mathrm{p})}\right)$ for $60 \mu \mathrm{s}$ and detected in heterodyne mode with a Nyquist bandwidth of $11.48 \mathrm{kHz}$. Trapping dc potential was varied from 0.1 to $10 \mathrm{~V}$.

For determination of the shift in ICR orbital frequency of $\mathrm{C}_{6} \mathrm{H}_{6}^{+}$as a function of excitation voltageduration product, we held the trapping potential at 1 $V$, and excited the ions in either of two ways: (1) excitation if amplitude was varied from 4.75 to 30.0 $\mathrm{V}_{\langle\mathrm{p}-\mathrm{p})}$ (or from 5.98 to $26.74 \mathrm{~V}_{(\mathrm{p}-\mathrm{p})}$ for the cubic trap) at a fixed excitation period of $80 \mu \mathrm{s}$ (or $100 \mu \mathrm{s}$ in the cubic trap); or (2) excitation period was varied from 60 to $210 \mu \mathrm{s}$ (or from 60 to $240 \mu \mathrm{s}$ for the cubic trap) at a fixed excitation amplitude of $16.87 V_{(\mathrm{p}-\mathrm{p})}$ (or 11.94 $V_{(\mathrm{p}-\mathrm{p})}$ for the cubic trap). Vacuum chamber pressure was $0.3 \times 10^{-8}$ torr. The peak shape of the magnitude-mode FT/ICR signal for hyperbolic and cubic traps was also compared as a function of rf excitation amplitude at fixed excitation period.

For determination of magnitude-mode FT/ICR relative mass spectral peak heights of perfluorotri- $n$ butylamine (PFTBA) ions as a function of relative ICR orbital radius, ions were produced by electron impact (70-eV electron beam of $50-\mathrm{ms}$ duration at an emission 
current of $45 \mathrm{nA}$ ) of a gaseous sample at a pressure of $0.3 \times 10^{-8}$ torr. An ICR signal was excited by an if broadband frequency-sweep from 0 to $2.667 \mathrm{MHz}$ at a sweep rate of $700 \mathrm{~Hz} / \mu \mathrm{s}$, and acquired by direct sampling at $5.247 \mathrm{MHz}$ for $12.49 \mathrm{~ms}$ to yield $64 \mathrm{~K}$ time-domain data points, to which another $64 \mathrm{~K}$ zeros were added before discrete Fourier transformation. No apodization (windowing) was applied.

To test for space charge effects on ICR orbital frequency and signal amplitude, we held the pressure at $0.4 \times 10^{-8}$ torr and kept all experimental parameters constant except for the electron beam duration, which was varied from 5 to $2000 \mathrm{~ms}$ at an emission current of $40 \mathrm{nA}$ measured by a collector on the side of the trap remote from the electron filament.

Finally, for the accurate-mass determinations reported in Table 1, FT/ICR magnitude-mode spectral peak frequencies were determined by parabolic three-point interpolation and fitted to a calibration law of the form [36],

$$
m / q=A / \nu_{+}+B / \nu_{+}^{2}
$$

For $m / q$ in $u / e$ and $v_{+}$in $\mathrm{kHz}$, eq 10 gave best-fit values, $A=46755.87$ and $B=-2055.105$ (hyperbolic); $A=46741.78$ and $B=-16399.17$ (cubic). AIthough higher-order calibration laws [37, 38] and more elaborate peak-fitting algorithms [39-42] are available, eq 10 suffices for the present purpose of comparing hyperbolic and cubic traps.

Ions were produced by electron ionization $(50-\mathrm{V}$ electron beam for $20 \mathrm{~ms}$ at an emission current of 55 $\mathrm{nA}$ ). The ions were excited by a frequency-sweep from dc to $715 \mathrm{kHz}$ at an rf amplitude of $21.24 V_{(\mathrm{p}-\mathrm{p})}$ ' and detected in heterodyne mode at a reference frequency of $705.96 \mathrm{kHz}$ for $45.27 \mathrm{~ms}$ to yield $64 \mathrm{~K}$ timedomain data points, to which another $64 \mathrm{~K}$ zeros were added $[43,44]$ before discrete Fourier transformation. No apodization was applied.

\section{Results and Discussion}

\section{Effect of Trapping Voltage on ICR Orbital Frequency}

Although ICR frequency is invariant with ion position in a perfect quadrupolar potential, eq 9a shows that ICR orbital frequency nevertheless varies with trapping voltage. For trapped-ion masses much less than the "critical" mass of eq $8 \mathrm{c}$ ( $m_{\text {crit }} \approx 26,000 \mathrm{u}$ at $3.0 \mathrm{~T}$ for $1-V$ trapping potential), the square root in eq $9 a$ may be expanded in a Taylor series to first order to yield,

$$
\omega_{+} \approx \omega_{\mathrm{c}}-\frac{2 V_{\mathrm{T}}}{\mathrm{B}_{0}\left(r_{0}^{2}+2 z_{0}^{2}\right)}
$$

In other words, to first order, the ICR orbital frequency in a quadrupolar electrostatic trapping potential varies linearly with applied trapping voltage, $V_{\mathrm{T}}$. The derivative of eq 11 with respect to trapping voltage gives the (downward) "trapping shift" of ICR orbital frequency with increasing trapping voltage:

$$
\frac{\partial \omega_{t}}{\partial V_{\mathrm{T}}}=-\frac{2}{B_{0}\left(r_{0}^{2}+2 z_{0}^{2}\right)} \quad\left(\mathrm{rad} \mathrm{s}^{-1} / \mathrm{N}\right)
$$

or

$$
\frac{\partial \nu_{+}}{\partial V_{T}}=-\frac{1}{\pi B_{0}\left(r_{0}^{2}+2 z_{0}^{2}\right)} \quad(\mathrm{Hz} / \mathrm{V})
$$

Equation 12 predicts a frequency shift, $\partial v_{0} / \partial V_{\mathrm{T}}=$ $438.6 \mathrm{~Hz} / \mathrm{V}$, for our hyperbolic ion trap, in excellent

\begin{tabular}{|c|c|c|c|c|}
\hline & ICR frequency & True mass & Measured mass & Esror \\
\hline \multicolumn{5}{|c|}{ Hyperbolic ion trap } \\
\hline Ion & $(\mathrm{kHz})$ & (u) & (u) & $\{\mathrm{ppm}\}$ \\
\hline $\mathrm{CF}_{3}^{+}$ & 676.77342 & 68.99466 & 68.99463 & -0.5 \\
\hline $\mathrm{C}_{3} \mathrm{~F}_{\mathrm{E}}^{+}$ & 356.03583 & 130.99147 & 130.99173 & 1.9 \\
\hline $\mathrm{C}_{4} \mathrm{~F}_{9}^{+}$ & 212.60808 & 218.98508 & 218.98540 & 1.4 \\
\hline $\mathrm{C}_{5} \mathrm{~F}_{10} \mathrm{~N}^{+}$ & 176.21067 & 263.98656 & 263.98632 & -0.9 \\
\hline $\mathrm{C}_{8} \mathrm{~F}_{16} \mathrm{~N}^{+}$ & 112.03666 & 413.97698 & 413.97613 & -2.1 \\
\hline $\mathrm{C}_{9} \mathrm{~F}_{20} \mathrm{~N}^{+}$ & 92.23658 & 501.97059 & 501.96920 & -2.9 \\
\hline \multicolumn{5}{|c|}{ Cubic ion trap } \\
\hline $\mathrm{CF}_{3}^{+}$ & 677.40793 & 68.99466 & 68.99472 & 0.8 \\
\hline $\mathrm{C}_{3} \mathrm{~F}_{5}^{+}$ & 356.63343 & 130.99147 & 130.99106 & -3.1 \\
\hline $\mathrm{C}_{4} \mathrm{~F}_{9}^{+}$ & 213.18789 & 218.98508 & 218.98454 & -2.5 \\
\hline $\mathrm{C}_{5} \mathrm{~F}_{10} \mathrm{~N}^{+}$ & 176.78525 & 263.98656 & 268.98708 & 1.8 \\
\hline $\mathrm{C}_{8} \mathrm{~F}_{16} \mathrm{~N}^{+}$ & 112.60527 & 413.97698 & 413.97835 & 3.3 \\
\hline $\mathrm{C}_{9} \mathrm{~F}_{20} \mathrm{~N}^{+}$ & 92.80398 & 501.97059 & 501.97274 & 4.3 \\
\hline
\end{tabular}
agreement with our observed experimental result of $441 \mathrm{~Hz} / \mathrm{V}$ obtained from a plot (see Figure 3a) of ICR

Table 1. Fourier transform ICR mass calibration for perfluorotri-n-butylamine 

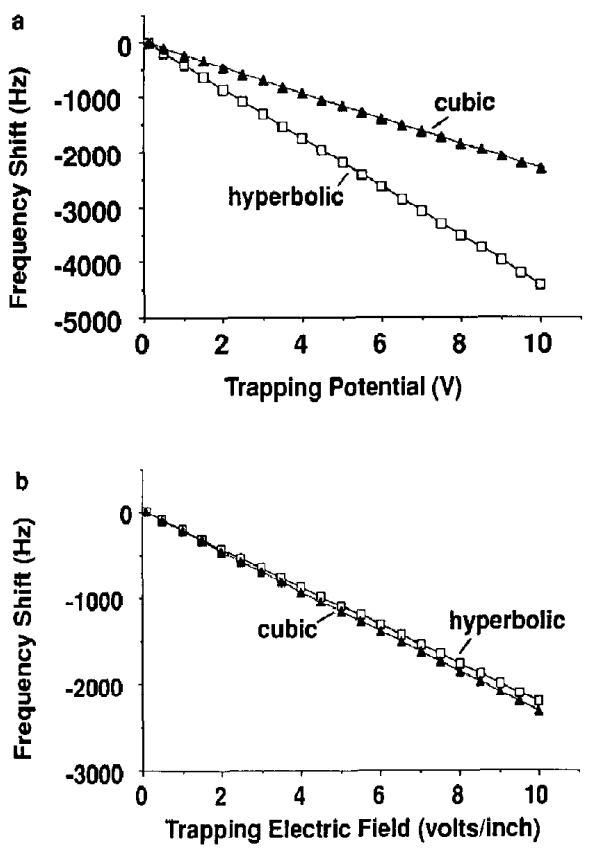

Figure 3. Experimental ICR orbital frequency shift (of $\mathrm{C}_{6} \mathrm{H}_{6}^{+}$at $3 \mathrm{~T}$ ) versus trapping voltage (a) or trapping electric field (b) in hyperbolic (0.5-in. separation between end cap electrodes) and cubic (1-in. trap plate separation) ion traps. The unshifted ICR orbital frequency, $599.06 \mathrm{kHz}$, is obtained by extrapolating a to zero trapping potential.

orbital frequency of $\mathrm{C}_{6} \mathrm{H}_{6}^{+}$versus trapping voltage at 3.0 T. A similar result was observed for a 1-in. cubic ion trap (Figure 3a), whose observed frequency shift of $231 \mathrm{~Hz} / \mathrm{V}$ agrees closely with the theoretical value $[22,25]$ of $223 \mathrm{~V} / \mathrm{Hz}$. Because the separation between the trapping electrodes of our hyperbolic trap is exactly half that of our cubic trap, the results are more readily compared by plotting ICR orbital frequency shift versus trapping electric field rather than trapping electrostatic potential difference (see Figure 3b). The close agreement between the two curves in Figure $3 \mathrm{~b}$ testifies to the accuracy of the quadrupolar approximation to the electrostatic field in both traps at relatively small ICR orbital radius.

\section{Effect of Radiofrequency Electric Field Excitation Amplitude-Duration Product on ICR Orbital Frequency}

As noted above, one of the most desirable features of the quadrupolar field of an infinitely extended hyperbolic trap is that the ICR orbital frequency is independent of ICR orbital radius. However, because the actual hyperbolic trap electrodes are not infinite in extent, an actual hyperbolic trap does not generate a purely quadrupolar potential, and the observed ICR orbital frequency therefore still varies somewhat with ICR orbital radius [35]. For single-frequency excitation of ions initially at rest on the $z$-axis, the postexcitation ICR orbital radius should be approximately proportional to the product of if electric field excitation amplitude, $V_{(p-p) r}$ and the excitation period, $T_{\text {excite }}$ [24]. Thus, we may test for the quadrupolar exactness of the hyperbolic trap potential by measuring the shift (if any) in ICR orbital frequency as a function of the product of $V_{(\mathrm{p}-\mathrm{p})}$ and $T_{\text {excite }}$.

The experimental results of Figure 4 indicate that the variation of ICR orbital frequency with ICR orbital radius is $\sim 15$ times smaller for the hyperbolic trap than for the cubic trap, over a wide range of ICR orbital radii. The residual frequency variation with radius in the hyperbolic trap may be due to: (1) the finite extent (and/or imperfect shape) of the trap electrodes, distorting the potential from a purely quadrupolar profile; (2) space charge effects; (3) inhomogeneity in magnetic field induction, and other nonlinearities discussed below. The measured ICR orbital frequency does drop off at very small ICR orbital radius for both hyperbolic and cubic traps; however, those measurements are least accurate because the measured signal current $[25,33]$ on the detector plates is small, and because space charge effects [45] are expected to be most significant at small radii at which the Lorentz magnetic force is relatively weak.

\section{Effect of Radiofrequency Electric Field Excitation Amplitude on FT/ICR Magnitude-Mode Spectral Peak Shape and Radial Ejection}

Figure 5 shows the FT/ICR magnitude-mode spectral peak shape as a function of relative ICR orbital radius (varied by changing the of electric field excitation
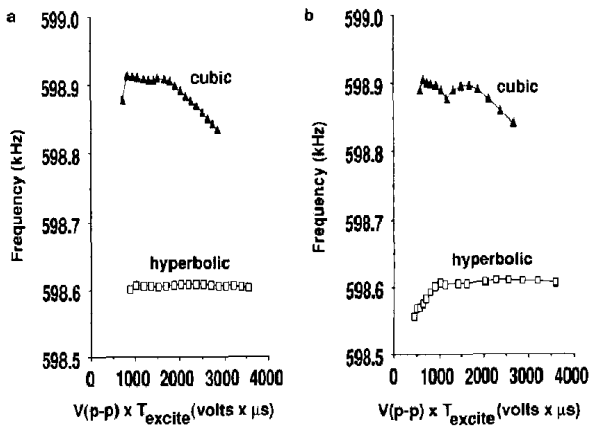

Figure 4. Experimental ICR orbital frequency in hyperbolic and cubic ion traps as a function of the product of single-frequency resonant rf electric field excitation amplitude and duration. (a) Radiofrequency electric field excitation period varied at constant If amplitude; (b) rf electric field excitation amplitude varied at constant excitation period. In either plot, the abscissa value is a measure of the relative ICR orbital radius. 

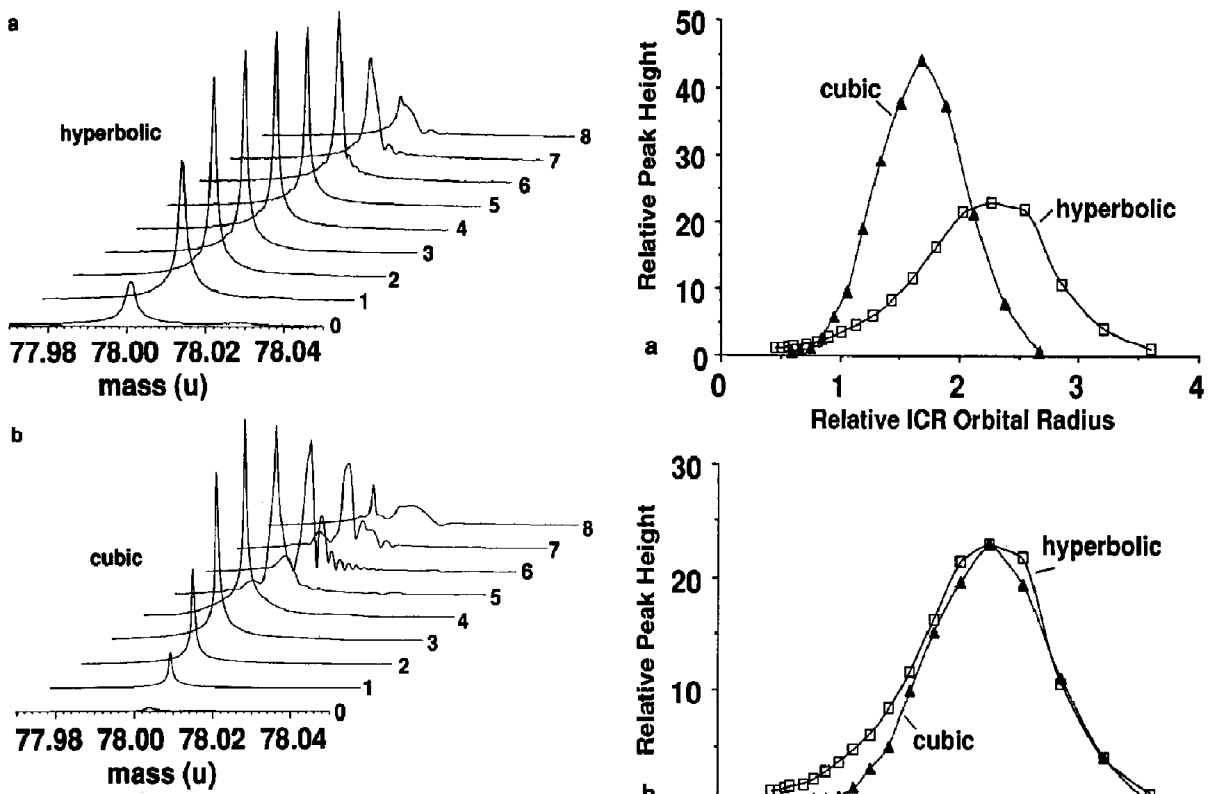

Figure 5. FT/ICR magnitude-mode spectra as a function of relative ICR orbital radius (varied by changing the if electric field excitation amplitude at constant excitation period). (a) Hyperbolic lon trap; (b) 1-in. cubic ion trap. In each case, the spectra are labeled according to the excitation if voltage attenuation, $A$ (in $\mathrm{dB}$ ), which is related to of peak-to-peak excitation voltage on our instrument according to the equation, $V_{(\mathrm{p}-\mathrm{p})}=30$ $\times 10^{-A / 20}$.

amplitude at constant excitation period). Note that the hyperbolic trap yields somewhat more uniform mass spectral peak shape than does the cubic trap, over a wide range of ICR orbital radius. The auxiliary peaks evident for both traps are due to a complex interplay of $z$-axis and cyclotron motion and have been analyzed $[46,47]$.

Figure 6a shows the measured onset of radial ejection as a function of relative ICR orbital radius, which is in turn proportional to the product of singlefrequency resonant if excitation voltage and duration. For the hyperbolic trap, longer and/or higher-amplitude excitation voltage is needed than for the cubic trap, because the excitation electrodes are (minimally) 1 in. apart (as in the cubic trap), but the trapping electrodes are separated by only $1 / 2$ in. and therefore the effective excitation if electric field is reduced. However, if we rescale the axes for the hyperbolic trap data to overlay the data for the cubic trap (see Figure $6 \mathrm{~b}$ ), we find that the detected signal versus ICR orbital radius behavior is quite similar for both traps. We conclude that hyperbolic and cubic traps exhibit similar radial ejection behavior, for the same rf electric field amplitude in both traps.

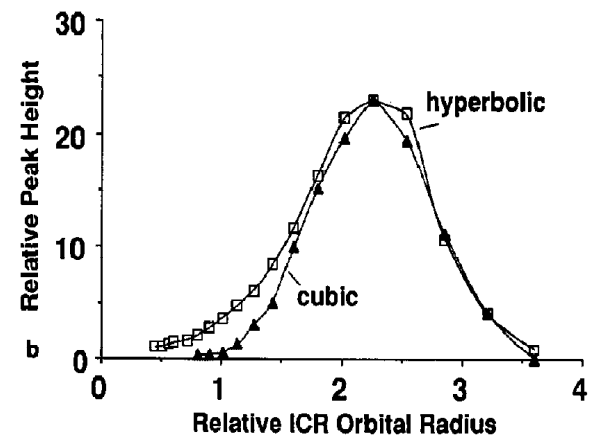

Figure 6. Radial ejection behavior in hyperbolic and cubic ion traps, illustrated by plots of experimental FT/ICR magnitudemode spectral relative peak height for $\mathrm{C}_{6} \mathrm{H}_{6}^{+}$ions versus relative ICR orbital radius (expressed as relative of electric field excitation amplitude-duration product). (a) Unscaled data. (b) Same data with both axes rescaled to account for different $\mathrm{rf}$ electric field produced by application of the same differential if voltage to the hyperbolic and cubic trap excitation electrodes and different signal strength in the two traps (see text).

It is perhaps at first surprising that the detected ICR signal from the hyperbolic trap does not drop off more suddenly following the onset of radial ejection (Figure 6a and $b$ ). The explanation likely lies in effects leading to loss of spatial and/or phase coherence in the ion packet during the excitation process. The preexcitation maximum radius of the ion packet is determined by the electron beam radius $(\sim 1 \mathrm{~mm})$, which is $\sim 10 \%$ of the minimum radius $(\sim 12 \mathrm{~mm})$ of the trap. Thus, any further spreading out of the ion packet will inhomogeneously broaden $[47,48]$ the FT/ICR mass spectral peak and reduce its height. Such effects could result from dephasing (at fixed ICR orbital radius) and/or increase in packet size, arising from a combination of space charge and/or nonlinear If excitation electric field, distribution in initial $z$ velocity, magnetic freld gradients, and nonquadrupolar static electric field (due to finite electrode dimensions and/or deviation of electrode shapes from perfectly smooth hyperboloids of revolution). For example, $z$-variation in the rf excitation electric field ampli- 
tude [49] leads to differential excitation and $z$-ejection (rather than radial ejection), whose effect varies with the preexcitation $z$-oscillation amplitude of an ion; thus, ions with different initial $z$-velocity will be $z$ ejected at different excitation levels, thereby broadening the radial ejection versus radius threshold. Moreover, because the detection process is nonlinear as well $[25,50]$, an increasing proportion of the detected signal appears at third (and higher-order) harmonics as ICR orbital radius increases, thereby producing a nonlinear variation of signal magnitude at the fundamental ICR orbital frequency with excitation rf amplitude-duration product, and thus broadening the radial ejection versus radius onset. Finally, the detected signal magnitude and frequency depend on ion preexcitation z-position, further complicating the analysis.

\section{Variation of FT / ICR Magnitude-Mode Relative Peak Height with ICR Orbital Radius}

For a spatially uniform if electric field excitation with uniform power at all frequencies, ions of all mass-tocharge ratios are excited to the same ICR orbital radius [51-53]. However, it is well known that $z$-inhomogeneity in the rf electric field excitation amplitude can lead to mass-dependent z-excitation (and even $z$-ejection) in ICR ion traps, because rf excitation applied across the transmitter electrodes has a signifcant rf electric field component in the $\pm z$-direction. Generally, the extent of $z$-ejection varies directly with ion preexcitation $z$-oscillation amplitude, and inversely with mass-to-charge ratio and applied trapping potential $[22,46,54]$. Thus, the FT/ICR mass spectral peak areas of low-mass ions typically decrease relative to those of high-mass ions, as ions are excited to larger ICR orbital radii. Also, z-ejection can be virtually eliminated by "shimming" the rf field to near-uniformity by addition of "guard" wires to a cubic trap [29-31].

Relative to the unshimmed cubic trap, the hyperbolic trap produces an even more anisotropic if electric field, which is strongest in the $z=0$ midplane. Therefore, z-ejection might be expected to pose a major problem. In Figure 7, we test for z-ejection by examining the dependence of FT/ICR magnitudemode mass spectral relative peak height (scaled to 100 for $\mathrm{CF}_{3}^{+}$at $69 u / e$ ) on relative ICR orbital radius. Figure $7 \mathrm{~b}$ shows that low-mass ions are preferentially lost (presumably by $z$-ejection) as ICR orbital radius increases. Comparison of Figure $7 a$ and $b$ shows that $z$-excitation and $z$-ejection are more severe in the hyperbolic trap than in the cubic trap.

\section{Effect of Number of Formed and Trapped Ions on ICR Signal Magnitude and Frequency}

Other parameters equal, the number of ions formed by electron ionization should be directly proportional to the electron beam duration. However, for the hy-
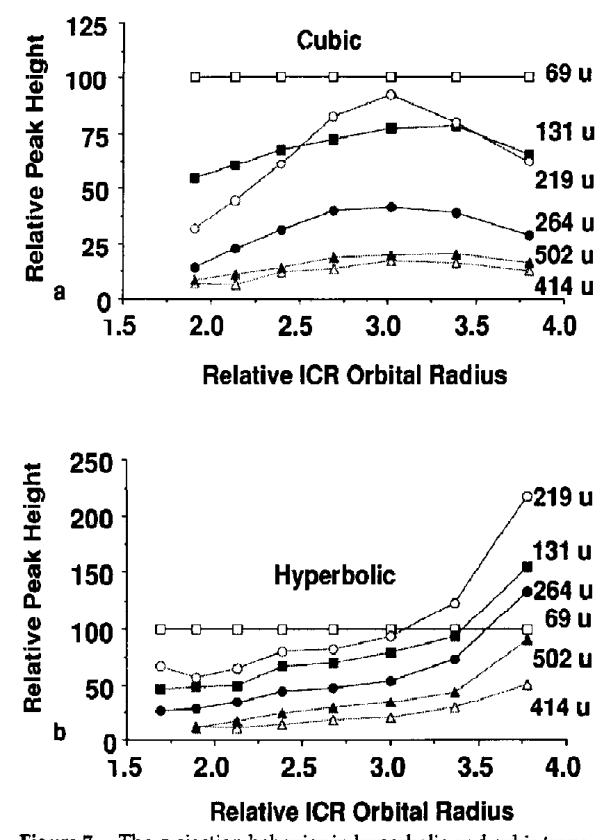

Figure 7. The z-ejection behavior in hyperbolic and cubic traps, illustrated as in Figure 6, except that the relative FT/ICR magnitude-mode spectral peak height for $\mathrm{CF}_{3}^{+}$ions at $m / z=69 u / e$ is scaled to 100 for all relative ICR orbital radii. (a) One-inch cubic trap; (b) hyperbolic trap. In each case, the abscissa represents the product of if peak-to-peak excitation voltage and excitation period, scaled as in Figure 6.

perbolic trap, the detected signal magnitude initially increases directly with number of formed ions, but eventually levels off to a near-constant value. Similar behavior has been reported for ions in a cubic trap [55]. Evidently, the number of ions which can be retained in any trap reaches a maximum, and any additionally formed ions leak out and are not detected. Hogan and Laude [56] have observed such effects with gated trapping experiments.

Figure 8 shows that the experimentally measured ICR orbital frequency of $\mathrm{C}_{6} \mathrm{H}_{6}^{+}$in both hyperbolic and cubic traps initially decreases and then levels off as the number of formed ions increases. The initial decrease in ICR orbital frequency is evidently due to increasing space charge with increasing number of trapped ions. The leveling effect is associated with the maximum ion-retaining capacity of the trap, as noted above.

\section{Mass Resolving Power}

We would expect that a hyperbolic trap should produce a narrower FT/ICR spectral peak than a cubic trap, since ions at different locations in the trap should 


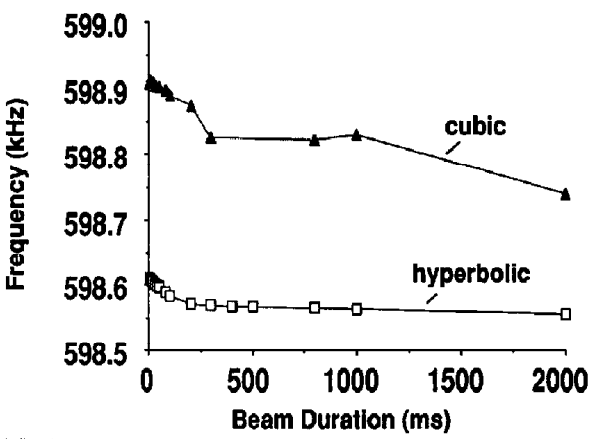

Figure 8. Variation of experimental ICR orbital frequency as a function of number of formed ions (as measured by the duration of an electron beam of fixed emission current).

have the same ICR orbital frequency in the near-perfect quadrupolar electrostatic trapping potential of the hyperbolic trap. In support of that prediction, Figure 9 shows an ultrahigh-resolution $(m / \Delta m=28.5$ million, for $\Delta m$ measured as full width at half-maximum peak height) FT/ICR magnitude-mode mass spectrum of $\mathrm{H}_{2} \mathrm{O}^{+}$(neutral pressure of $-1.1 \times 10^{-9}$ torr, with single-frequency on-resonance excitation ( $30 V_{(p-p)}$ at $2.607 \mathrm{MHz}$ for $95 \mu \mathrm{s}$ ), and detected in heterodyne mode at a bandwidth of $1.422 \mathrm{kHz}$ ). That performance was significantly higher than the best we were able to achieve with a cubic trap under comparable conditions.

\section{Mass Measurement Accuracy}

Given the more homogeneous peak shape from the hyperbolic trap, one might hope for higher mass measurement accuracy than with a cubic trap. Table 1 gives "best-effort" results under comparable conditions for mass calibration [36] accuracy over a wide

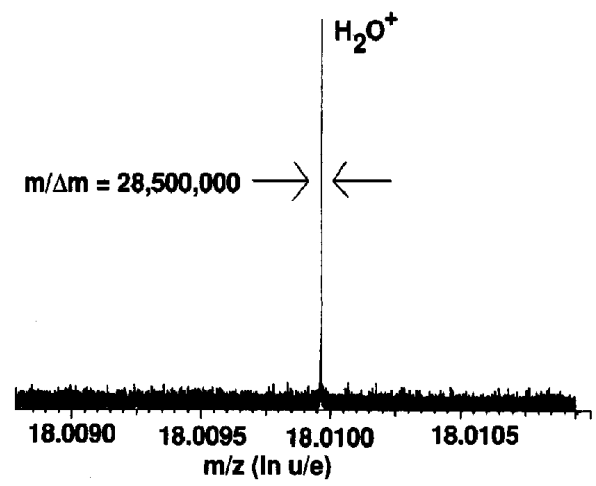

Figure 9. Ultrahigh-resolution FT/ICR mass spectrum of $\mathrm{H}_{2} \mathrm{O}^{+}$ obtained with the hyperbolic trap. mass range $(60 u / e \leq m / z \leq 600 u / e)$, for six relatively abundant ions from electron-ionized PFTBA. It appears that mass accuracy is about twice as high for the hyperbolic trap as for the cubic trap.

\section{Hyperbolic Trap Relative Dimensions}

It is perhaps worth noting that the most common hyperbolic trap is one for which $r_{0}=\sqrt{2} z_{0}$ [32]. However, a pure quadrupole potential can be generated by hyperbolic traps of any $r_{0} / z_{0}$ ratio [57]. The unique property of the $r_{0}=\sqrt{2} z_{0}$ design is simply that the hyperbolic end cap and ring electrodes approach their common asymptote symmetrically in that case [57]. In the present work, we employed a hyperbolic trap with $r_{0}=2 z_{0}$.

\section{Other Configurations and Operating Modes for the Hyperbolic Trap}

Three ICR excitation and detection modes have been applied in various combinations to hyperbolic traps. In parametric excitation (or detection), an $\mathrm{rf}$ voltage is applied (or detected) between the end caps and ring electrode-in this mode, ions must initially be uniformly displaced away from the z-axis. In dipolar excitation (or detection), as in this work, an $\mathrm{rf}$ voltage is applied (or detected) between two opposed segments of a ring electrode which has been sliced into four (or more) sectors. In quadrupolar excitation (or detection), an rf voltage of one sign is applied (or detected) between two opposed ring quadrants, while an identical rf voltage of opposite sign is applied (or detected) between two other opposed ring quadrants orthogonal to the first pair.

In the first application of parametric excitation and detection to a hyperbolic trap [58], it was found that more ions could be trapped and detected than with dipolar excitation/detection in a cubic trap, presumably because ions in the parametric case may be distributed around the entire circumference of a given isopotential surface, rather than (as in dipolar operation) having to be spatially localized in a one-dimensional coherent ion packet. Rempel et al. [58] formed ions off-axis initially by use of an off-axis electron beam. Alternatively, Schweikhard et al. [59] showed that ions may be formed on-axis, and then moved off-axis by magnetron excitation, followed by parametric excitation and dipolar detection.

Yet another approach is the use of a ring electrode cut into four (or eight) sections, in which quadrupolar excitation [60] and quadrupolar detection are employed [61-63]. For example, Schweikhard et al. [61] added the signals from two opposed ring quadrants to produce a detected signal with the same character (but half the magnitude) as the full quadrupolar signal. Schweikhard et al. [62] subsequently showed that the quadrupolar operation could yield a detected sig- 
nal at frequency, $\left(\omega_{+}+\omega_{-}\right)=\omega_{c}$. In other words, it is possible to detect a signal whose ICR frequency is unshifted by the electrostatic trapping field. The theory of the quadrupolar experiment has recently been developed in more detail [63].

\section{Upper Mass Limit}

The upper mass limit, $m_{\text {crit }}$ (see eq $8 \mathrm{c}$ ) for singly charged ions in FT/ICR mass spectrometry is determined by the dimensions of the ion trap and the trapping potential. In this respect, the hyperbolic trap offers no theoretical improvement over a cubic trap, and is theoretically inferior to a screened trap [28].

\section{Conclusion}

In summary, comparison of hyperbolic and cubic ion traps offers a direct probe of the effect of nonquadrupolar electrostatic trapping field on FT/ICR mass spectrometry performance. From the various prior and present experimental and theoretical results discussed above, it appears that the hyperbolic trap offers several advantages over cubic or cylindrical traps: improved mass resolving power and more symmetrical FT/ICR mass spectral peak shape for highresolution experiments, improved mass accuracy for wide-range mass spectra, and (depending on choice of excitation and detection mode) higher dynamic range and/or elimination of frequency shifts due to trapping potential. However, z-ejection (and hence the variation of relative FT/ICR mass spectral peak heights of ions of different mass-to-charge ratio as a function of ICR orbital radius) is more pronounced in the hyperbolic than in the cubic trap. Moreover, the trapping field limits the maximum theoretical massto-charge ratio in all but the screened trap [28]. Further systematic and direct experimental comparisons between various trap configurations and operating modes should provide additional insight into spectral distortions resulting from inhomogeneity and/or nonlinearity in the trapping, excitation, and detection processes in FT/ICR mass spectrometry.

\section{Acknowledgments}

The authors thank L. Schweikhard for helpful discussions. This work was supported by grants (to Dr. Marshall) from the National Science Foundation (CHE-90-21058) and The Ohio State University.

\section{References}

1. Asamoto, B. Spectroscopy 1988, 3, 38-46.

2. Buchanan, M. V., Ed. Fourier Transform Mass Spectrometry: Evolution, Innovation, and Applications; ACS Symp. Ser. 1987; 359 .

3. Chiarelli, M. P.; Gross, M. L. In Analytical Applications of Spectroscopy; C. S. Creaser; A. M. C. Davies, Eds. Royal Society of Chemistry: London, 1988; pp 263-273.
4. Cody, R. B. Analysis 1988, 16, 30-36.

5. Freiser, B. S. In Techniques for the Study of lon Molecule Reactions. J, M. Farra, W. H. Jr. Saunders, Eds.; Wiley: New York, 1988; Vol. 20; pp 61-118.

6. Freiser, B. S. Chemtracts-Anal. Phys. Chem. 1989, 1, 65-109.

7. Gord, J. R.; Freiser, B. S. Anal. Chim. Acta 1989, 225, 11-24.

8. Hanson, C. D.; Kerley, E. L.; Russell, D. H. In Treatise on Analytical Chemistry, 2nd ed.; J. D. Winefordner, Ed.; Wiley: New York, 1988; Vol. 11, pp 117-187.

9. Lubman, D. M.; Ed. Lasers in Mass Spectrometry; Oxford University Press: New York, 1990; chapters 7, 11-15.

10. Marshall, A. G. Acc. Chem. Res. 1985, 18, 316-322.

11. Marshall, A. G.; Grosshans, P. B. Anal. Chem. 1991, 63, 215A-229A.

12. Nibbering, N. M. M. Acc. Chem. Res. 1990, 23, 279-285.

13. Russell, D. H. Mass Spectrom. Rev. 1986, 5, 167-189

14. Sharpe, P.; Richardson, D. E. Coord. Chem. Rev. 1989, 93, $59-85$.

15. Wanczek, K.-P. Int. J. Mass Spectrom. Ion Processes 1989, 95, $1-38$.

16. Wilkins, C. L.; Chowdhury, A. K.; Nuwaysir, L. M.; Coates, M. L. Mass Spectrom. Rev. 1989, 8, 67-92.

17. Comisarow, M. B. Adv. Mass Spectrom. 1980, 8, 1698-1706 Comisarow, M. B. Int J. Mass Spectrom. Ion Phys. 1981, 37, 251-257.

18. Comisarow, M. B.; Marshall, A. G. Chem. Phys. Lett. 1974, 25, 282-283. Comisarow, M. B.; Marshall, A. G. Chem. Phys. Lett. 1974, 26, 489-490.

19. Hunter, R. L.; Sherman, M. G.; McIver, R. T. Jr.; Int. J. Mass Spectront., Ion Phys. 1983, 50, 259-274

20. Comisarow, M. B.; Marshall, A. G. U.S. Patent No. 3,937,955, issued Feb. 14, 1976.

21. Lee, S. H.; Wanczek, K-P.; Hartmann, H. Adv. Mass Spectrom. 1980, 8, 1645.

22. Kofel, P.; Allemann, Mi; Kellerhals, H. P, Wanczek, K.-P. Int. J. Mass Spectrom. Ion Processes 1986, 74, 1-12.

23. Elkind, J. L.; Weiss, F. D.; Alford, J. M.; Laaksonen, R. T, Smalley, R. E. J. Chem. Phys. 1988, 88, 5215-5224.

24. Grosshans, P. G.; Marshall, A. G. Int. J. Mass Spectrom. Ion Processes 1990, 100, 347-379.

25. Grosshans, P. B.; Shields, P. J.; Marshall, A. G. J. Chem. Phys. 1991, 94, 5342-5352.

26. Yang, S. S.; Rempel, D. L.; Gross, M. L. In Proceedings of the 36th American Society for Mass Spectrometry Annual Conference, San Francisco, June 1988, pp 586-587.

27. Naito, Y.; Inoue, M. In Proceedings of the 36th American Society Mass Spectrometry Annual Conference, San Francisco, June 1988, pp 608-609.

28. Wang, M.; Marshall, A. G. Anal. Chem. 1989, 61, 1288-1293.

29. Sommer, H.; Thomas, H. A.; Hipple, J. A. Phys. Rev. 1951, 82, 697-702.

30. Wang, M,; Marshall, A. C. Anal. Chem. 1990, 62, 515-520.

31. Hanson, C. D.; Castro, M. E.; Kerley, E. L. Russell, D. H, Anal. Chem. 1990, 62, 520-526.

32. Brown, L. S.; Gabrielse, G. Rev. Mode Phys. 1986, 58, 233-311.

33. Comisarow, M. B. J. Chem. Phys. 1978, 69, 4097-4101.

34. van Dyck, R. S., Jr.; Schwinberg, P. B. Phys. Rev. Lett. 1981, 47, 395-398.

35. Wang, M.; Ledford, E. B. Jr.; Marshall, A. G. Presented at FACSS XIV Annual Meeting, Detroit, MI, Oct. 1987, Abstract 43.

36. Ledford, E. B. Jr.; Rempel, D. L.; Gross, M. L. Anal. Chem. 1984, 56, 2744-2748.

37. Wang, M.; Marshall, A. G. Int. I. Mass Spectrom. Ion Processes 1988, 86, 31-51. 
38. Smith, M. J. C. Presented at 2nd American Society for Mass Spectrometry, Sanibel Conference, Sanibel Island, FL, January 1990.

39. Giancaspro, C.; Comisarow, M. B. Appl. Spectrosc. 1983, 37, $153+166$.

40. Serreqi, A.; Comisarow, M. B. Appl. Spectrosc. 1987, A10, 288-298.

41. Keefe; C. D.; Comisarow, M. B. Appl. Spectrosc. 1990, 44, 600-613.

42. Verdun, F. R.; Giancaspro, C.; Marshall, A. G. Appl. Spectrosc. 1988, 42, 715-721.

43. Comisarow, M. B.; Melka, J. D. Anal. Chem, 1979, 51, 2187-2207.

44. Liang, Z.; Marshall, A. G. Appl. Spectrosc. 1990, 44, 766-775.

45. Wang, T.-C. L.; Marshall, A. G. Int. I. Mass Spectrom. Ion Processes 1986, 68, 287-301.

46. Huang, S. K.; Rempel, D. L.; Gross, M. L. Int. l. Spectrom. Ion Processes 1986, 72, 15-31.

47. Rempel, D. L.; Grese, R. P.; Gross, M. L. Int. J. Mass Spectrom. Ion Processers 1990, 100, 381-395.

48. Comisarow, M. B. In Lecture Notes in Chemistry; Ion Cyclotron Resonance Spectrometry II; H. Hartmann, K.P. Wanczek, Eds.; Springer-Verlag: Berlin, 1982; Vol. 31, pp 484-513.

49. Dunbar, R. C. Int. J. Spectrom. Ion Processes 1984, 56, 1-9.

50. Grosshans, P. B.; Marshall, A. G. Int. I. Mass Spectrom. Ion Processes 1991, 107, 49-81.
51. Beauchamp, J. L. I. Chem. Phys. 1966, 46, 1231-1243.

52. Hearn, B. A.; Watson, C. H.; Baykut, G.; Eyler, J. E. Int. J. Mass. Spectrom. Ion Phys. 1990, 95, 299-316.

53. Wang, M.; Marshall, A. G. Int. J. Mass Spectrom. Ion Processes 1990, 100, 323-346.

54. Allemann, M.; Kofel, P.; Kellerhals, Hp.; Wanczek, K.-P. Int. J. Mass Spectrom. Ion Processes 1987, 75, 47-54.

55. Rempel, D. L.; Huang, S. K.; Gross, M. L. Int. J. Mass. Spectrom. Ion Processes 1986, 70, 163-184.

56. Hogan, J. D.; Laude, D. A., Jr. I. Am. Soc. Mass Spectrom. 1990, 1, 431-439.

57. Knight, R. D. Int. J. Mass Spectrom. Ion Phys. 1983, 51, 127-131.

58. Rempel, D. L.; Ledford, E. B. Jr.; Huang, S. K.; Gross, M. L. Anal. Chem. 1987, 59, 2527-2352.

59. Schweikhard, L.; Lindinger, M.; Kluge, H.-J. Rev. Sci. Instrum. 1990, 61, 1055-1058.

60. Becker, St.; Bollen, G.; Kern, F.; Kluge, H.-J.; Moore, R. B.; Savard, C.; Schweikhard, L.; Stolzenberg, H. Int. I. Mass Spectrom. Ion Processes 1990, 99, 53-57.

61. Schweikhard, L.; Blundschling, M.; Jertz, R.; Kluge, H.-J Rev. Sci. Instrum. 1989, 60, 2631-2634.

62. Schweikhard, L.; Lindinger, $M_{\text {; }}$ Kluge, H.-J. Mass Spectrom. Ion Processes 1990, 98, 25-33.

63. Schweikhard, L. Int. J. Mass Spectrom. Ion Processes 1991, $107,281-292$ 